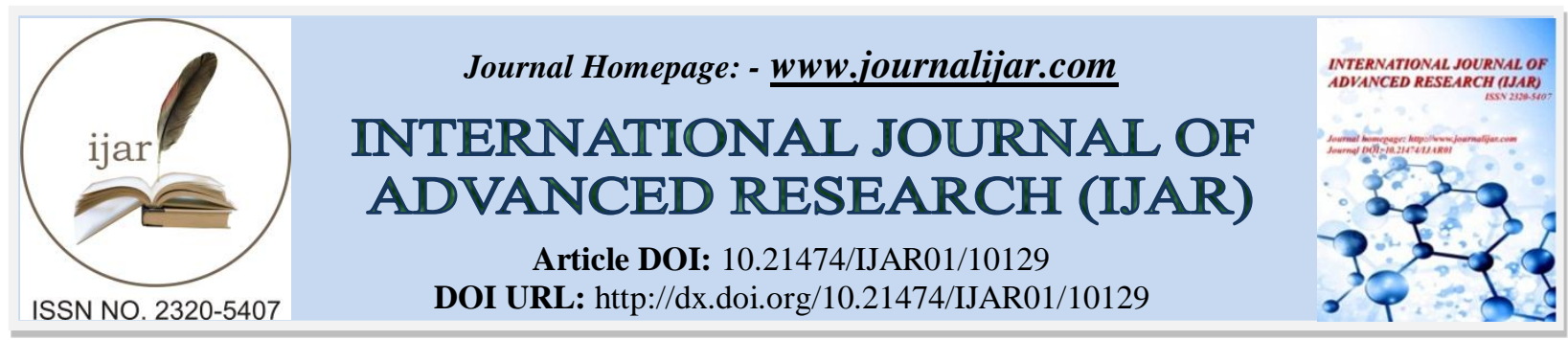

RESEARCH ARTICLE

\title{
BILATERAL LICHTENSTEIN TENSION FREE REPAIR OF INGUINAL HERNIA AND TESTICULAR FUNCTION; A PROSPECTIVE CLINICAL TRIAL.
}

\author{
Ahmed M. El Teliti, Mostafa M.Khairy and Hazem Nour. \\ General surgery department.Zagazig university, Egypt.
}

\section{Manuscript Info}

Manuscript History

Received: 01 October 2019

Final Accepted: 03 November 2019

Published: December 2019

Key words:-

Lichtenstein Repair, Inguinal Hernia,

Testicular Function, Semen Analysis.

\begin{abstract}
Background:Lichtenstein tension free repair of inguinal hernia a very effective repair but fibrosis generated around the mesh prosthesis may be hazardous to the vas deference and testicular blood supply thus impairing testicular function especially if done on both sides.

Patient and methods:78 patients with bilateral inguinal hernia recruited in this prospective trial for evaluation of the effect of bilateral Lichtenstein tension free repair of inguinal hernia on testicular function we held comparison between preoperative and 6 months postoperative findings of; testicular volume, testicular blood flow (resistive index) semen analysis and free testosterone level as primary outcome and surgical site occurrences and recurrence rate as secondary outcome.

Results: mean Age, BMI, Operative time were $33.1 \pm 8.8$ years , 27.6 \pm 3.1 , and $98.4 \pm 11.4$ respectively, majority of hernia presentation was indirect hernia, we got 2 cases of chronic pain, one case of hydrocele and no recurrences, regarding testicular volume, resistive index, semen analysis and testosterone level noticed non-significant differences between pre and post repair results.

Conclusion:In regard to testicular function, bilateral Lichtenstein tension free repair of inguinal hernia is an effective safe method, especially if laparoscopic repair is not available.
\end{abstract}

Copy Right, IJAR, 2019,. All rights reserved.

\section{Introduction:-}

Inguinal hernia is a common surgical disease with life time risk about $27 \%(1)$, inguinal hernia repair is one of the most frequent operations carried out by general surgeons, nowadays more than 1 million repair is carried out each year(2), a part from laparoscopic repair, Lichtenstein mesh repair (LMR) is the most effective method of hernia repair as it carries the least recurrence rate, accepted post-operative pain tolerance and postoperative complications rate $(3,4,5)$, the principle of (LMR) is the formation of fibro-elastic mesh complex that reinforce the hernia defect site and helps preventing recurrence of the hernia $(3,6)$, the intense fibro-elastic tissue formation around the mesh prosthesis entangles the surrounding structures, of these structure the vas deference and its blood supply and the testicular artery which may be entangled in the fibrosis affecting the testicular perfusion and the testicular functions $(7,8)$, testicular functions may be affected in another ways after (LMR) as if the testicular artery or the vas deference were injured by diathermy, or accidentally torn out such situations proved rare in some literature (9), the risk of postoperative testicular hypo function increases in bilateral cases and with previous groin hernia surgery (10).Here in this study our objective is to evaluate the effect of bilateral (LMR) on testicular function.

Corresponding Author:-Ahmed M. El Teliti.

Address:-General surgery department.Zagazig university, Egypt. 


\section{Patients and Methods:-}

This study is prospective cohort study, carried out in the time between August 2017 and March 2019 on 78 male patients with bilateral inguinal hernia undergoing bilateral Lichtenstein mesh repair (LMR). Patients included in this study were male patients aged above 18 years old, patients excluded were those with ASA class 3 and 4, those with previous inguinal hernia, varicocele or testicular surgery, undescended testis, known testicular hypo-function, sexual inactivity, advanced renal, cardiac or liver diseases.

The primary outcome of this study is the effect of bilateral hernia repair on testicular function detected by testicular duplex study, (testicular volume, and testicular blood flow by calculation of resistive index), semen analysis and serum testosterone assay. The secondary outcome is the postoperative surgical site occurrences and recurrence rate within the follow up time

\section{All patients were informed and consented before the study:}

This study was approved by the local ethical committee and the institutional review board (IRB) of our hospital. All patients of the study were subjected to thorough history taking, clinical examination of the groin and scrotum, abdominal ultrasound, inguino-scrotal colored duplex for, confirmation of bilateral hernia, measurement of testicular volume and testicular perfusion by peak systolic velocity (PSV) and end diastolic velocity (EDV), and resistive index (RI), hormonal assay as free testosterone levels, and computer assisted semen analysis (CASA) semen analysis. All patients received prophylactic third generation cephalosporin $1 \mathrm{gm}$ intravenous injection just before surgery, then they underwent bilateral (LMR) under spinal anesthesia by the same group of surgeons, patients were followed up in the outpatient clinics in the first for operative week for local wound complications as surgical site infections, hematoma and seroma formation, and later on for chronic groin pain, after six months patient are subjected to duplex study of the testis, serum testosterone together with semen analysis.

\section{Principles of Lichtenstein tension free repair:}

Under spinal anesthesia, in the supine position, after skin preparation and patient draping inguinal incision done just one finger breadth above the medial 3 fifths of the inguinal ligament, the inguinal canal was opened by incising the external oblique apponeurosis in the direction parallel to that of the spermatic cord which is dissected from the surroundings with preservation of the ilioinguinal nerve, the floor of the inguinal canal was inspected for the presence of direct inguinal hernia and the cord coverings were dissected and cord explored for the presence of indirect inguinal hernia, in oblique hernia after identification of the hernial sac it was transfixed at the neck and the distal part was excised, we used intraoperative Doppler examination of the spermatic cord for identification the testicular artery, sometimes we used a magnifying loop, the deep ring was narrowed by non-absorbable suture to admit the tip of the little finger, in the presence direct hernia a purse string suture was taken around the neck of the sac and tied after sac invagination, fascia transversalis plication done by polypropylene suture as per need, a polypropylene mesh sheet was prepared and tailored to the area of the posterior wall of inguinal canal and fixed in position by non-absorbable suture, hemostasis achieved and suction drain inserted as per need.

Postoperative analgesia in the form of diclofenac sodium intramuscular injection was given as per need; patients were discharged after 24 hours.

All the preoperative parameters and follow up data were collected, properly analyzed, and compared using SPSS program $20^{\text {th }}$ edition.

The investigators have no conflict of interest and no external funding.

\section{Results:-}

A cohort of 78 male patients with bilateral inguinal hernia was recruited in this study, The mean age ranged between 18 and 65 years with mean age 33.1 years and standard deviation (SD) 8.8, BMI range was 20.5 to 36.1 with mean 27.6 \pm SD 3.1, hernia on right side was indirect in53, direct in 12 and both types were present 13 cases, on the left side indirect hernia was present in 51 cases, direct hernia in 15 cases and both types in 12 cases, the mean operative time was $98 \pm$ SD 11.4 minutes, as presented in (table 1)

Table 1:- Demographic data and operative time. 


\begin{tabular}{|c|c|c|c|}
\hline \multicolumn{2}{|l|}{ Age } & $18-65$ years & $33.1 \pm 8.8$ years \\
\hline \multicolumn{2}{|l|}{ BMI } & $20.5-36.1$ & $27.6 \pm 3.1$ \\
\hline \multirow{2}{*}{\multicolumn{2}{|c|}{ Operative time }} & $68-115$ minutes & $98.4 \pm 11.4$ \\
\hline & & left & Right \\
\hline \multirow[t]{3}{*}{ Hernia type } & Indirect & 51 & 53 \\
\hline & Direct & 15 & 12 \\
\hline & Both & 12 & 13 \\
\hline
\end{tabular}

As associated co morbidity we got 4 diabetic, 3 hypertensive and one bronchial asthma patients.

LMR was completed in all cases without any intraoperative complications, during the early follow up time we have 2 cases $(2.6 \%)$ of groin pain that last for 6 weeks, one case (1.3\%) developed hydrocele on the left side .

The primary outcome of this study obtained by comparing testicular volume, resistive index (RI), free testosterone level, and semen analysis before hernia repair and 6 months after hernia repair as presented in (table 2).Comparison between pre and postoperative scrotal duplex study shows non-significant difference regarding testicular size and resistive index. Pre and post-operative values of the spermiogram shows non-significant differences regarding average sperm count and rate of progressive motility, also there was a non-significant difference between pre and post-operative testosterone levels.

As a secondary outcome we have got no recurrences in the follow up time.

Table 2:- Post-operative outcome

\begin{tabular}{|l|l|l|l|l|}
\hline \multicolumn{2}{|l|}{} & preoperative & postoperative & p-value \\
\hline Testicular volume cm3 & Right & $17.8 \pm 1.03$ & $18.1 \pm 0.98$ & NS \\
\cline { 2 - 5 } & left & $18.6 \pm 1.1$ & $18.9 \pm 1.05$ & NS \\
\hline Resistive index (RI) & $0.75 \pm 0.07$ & $0.73 \pm 0.08$ & NS \\
\hline Sperm count(million $\backslash \mathrm{ml}$ ) & $53.5 \pm 9.3$ & $48.6 \pm 8.7$ & $\mathrm{NS}$ \\
\hline Rate of progressive motility \% & $51.64 \pm 2.29$ & $51.4 .1 \pm 2.1$ & $\mathrm{NS}$ \\
\hline Free testosterone level ng $\backslash \mathrm{ml}$ & $16.53 \pm 3.71$ & $15.93 \pm 3.65$ & $\mathrm{NS}$ \\
\hline
\end{tabular}

\section{Discussion:-}

Mesh repair is the standard treatment of inguinal hernia but, the fibroblastic reaction and the inflammatory process around mesh prosthesis lead to mesh shrinkage up to $60 \%$ of its size (11), this shrinkage may entangle the vas deference and testicular blood vessels leading to testicular ischemia that is reflected clinically by decrease in testicular volume and function.

The primary outcome of this study is changes in testicular functions, blood supply and testicular volume, through the short term follow up of patients we noted that bilateral LMR of inguinal hernia didn't affect testicular blood flow by calculation of resistive index of both sides, this in keeps with other studies $(10,12,13,14)$, but some studies $(15,16$, 17) reported different results, this controversy may come from heterogeneity of patient's response to mesh prosthesis.

Testicular volume in turn showed non-significant difference between preoperative and post-operative findings, Taylor et al (13) and LimaNeto et al (15) noted that contraction of mesh following repair of inguinal hernia doesn't affect testicular size but Homonnai et al (18) found that $14.4 \%$ of patient have had testicular atrophy following inguinal hernia mesh repair.

In our study we found no statistically significant difference between the pre $\&$ postoperative spermiogram regarding sperm concentration and rate of progressive sperm motility the same was reported by Aydede et al (19).

Regarding testosterone level we noted non-significant differences between pre and postoperative measurements these findings could be expected as long as we have got no changes in testicular size and blood flow. 
The recurrence rate can't be judged properly as the follow up time is relatively short. This study is limited by relatively short follow up time.

\section{Conclusion:-}

In regard to testicular function, bilateral Lichtenstein tension free repair of inguinal hernia is an effective safe method, especially if laparoscopic repair is not available

\section{References:-}

1. Gould, J. (2008). Laparoscopic versus open inguinal hernia repair. Surgical Clinics of North America, 88(5), 1073-1081.

2. Amid PK, Friis E, Horeyseck G, Kux M (1999) A multi-center experience with 6764 Lichtenstein tension-free hernioplasties. Hernia 3:47-8

3. Lichtenstein IL, Shulman AG, Amid PK, MontXor MM (1989) the tension-free hernioplasty. Am J Surg 157:188-193

4. Hubner M, Vuilleumier H, Demartines N (2008) Sexual impairment due to mesh plug hernia repair? ANZ J Surg 78:213-219

5. Miller, H. J. (2018). Inguinal Hernia: Mastering the Anatomy. Surgical Clinics of North America, 98(3), 607621.

6. 6.Dilek, O. N. (2014). Hernioplasty and testicular perfusion. Springerplus, 3(1), 107.

7. Khan LR, Kumar S, Nixon SJ. (2006). Early results for new lightweight mesh in laparoscopic totally extraperitoneal inguinal hernia repair. Hernia. 2006 Aug; 10(4):303-8.

8. Shin D, Lipshultz LI, Goldstein M, Barme GA, Fuchs EF, Nagler HM, McCallum SW, Niederberger CS, Schoor RA, Brugh VM, Honig SC (2005) Herniorrhaphy and polypropylene mesh causing inguinal vassal obstruction: a preventable cause of obstructive azoospermia. Ann Surg 241(4):553-558

9. Fitzgibbons RJ (2007). The mesh and the spermatic cord. In: Schumpelick V, Fitzgibbons RJ (eds) Recurrent Hernia. Springer, Heidelberg, pp 333-337.

10. Turgut, A. T., Ölçücüoğlu, E., Turan, C., Kiliçoğlu, B., Koşar, P., Geyik, P. Ö., \& Dogra, V. (2007). Preoperative ultrasonographic evaluation of testicular volume and blood flow in patients with inguinal hernias. Journal of Ultrasound in Medicine,26(12), 1657-1666.

11. Amid PK (1997) Classification of biomaterials and their related complications in abdominal wall hernia surgery. Hernia 1:15-21

12. Beddy P, Ridgway PF, Geoghegan T, Peirce C, Govender P, Kean FB, Torreggiani WC, Conlon KE (2006) Inguinal hernia repair protects testicular function: a prospective study of open and laparoscopic herniorraphy. Am Coll Surg 203(1):17-23

13. Taylor SG, Hair A, Baxter GM, O'Dwyer PJ (2001) Does contraction of mesh following tension free hernioplasty eVect testicular or femoral vessel blood Xow? Hernia 5:13-15

14. Pepe P, Aragona F (2007) Testicular ischemia following mesh hernia repair and acute prostatitis. Ind J Urol 23(3):323-325

15. Lima Neto EV, Goldenberg A, Juca MJ (2007) Prospective study on the eVects of a polypropylene prosthesis on testicular volume and arterial Xow in patients undergoing surgical correction for inguinal hernia. Acta Cir Bras 22(4):226-271.

16. Zieren J, BeyersdorV D, Beier KM, Muller JM (2001) Sexual function and testicular perfusion after inguinal hernia repair with mesh. Am J Surg 181:204-206

17. Ramadan SU, Gokharman P, Tuncbilek I, Ozer H, Kosar P, Kosar U (2007) Does mesh/perimesh Wbrosis have an eVect on testicular Xow in patients with repaired inguinal hernia? Utraschall Med 28 (abstract)

18. Homonnai ZT, Fainman N, Paz GF, David MP (1980) Testicular function after herniotomy. Herniotomy and fertility. Andrologia 12:115-120

19. Aydede, H., Erhan, Y., Sakarya, A., Kara, E., Ilkgul, Ö, \& Can, M. (2003). Effect of mesh and its localization on testicular flow and spermatogenesis in patients with groin hernia. Acta Chirurgica Belgica, 103(6), 607-610. 